\title{
Endogenous entry in lowest-unique sealed-bid auctions
}

\author{
Harold Houba • Dinard van der Laan • \\ Dirk Veldhuizen
}

(C) The Author(s) 2010. This article is published with open access at Springerlink.com

\begin{abstract}
Lowest-unique sealed-bid auctions are auctions with endogenous participation, costly bids, and the lowest bid among all unique bids wins. Properties of symmetric NEs are studied. The symmetric NE with the lowest expected gains is the maximin outcome under symmetric strategies, and it is the solution to a mathematical program. Comparative statics for the number of bidders, the value of the item and the bidding cost are derived. The two bidders' auction is equivalent to the HawkDove game. Simulations of replicator dynamics provide numerical evidence that the symmetric NE with the lowest expected gains is also asymptotically stable.
\end{abstract}

Keywords Sealed-bid auction · Evolutionary stability · Endogenous entry · Maximin

JEL Classification $\quad \mathrm{D} 44 \cdot \mathrm{C} 72 \cdot \mathrm{C} 73 \cdot \mathrm{C} 61 \cdot \mathrm{L} 83$

This article expresses the personal views and opinions of the authors. Please note that SNS Reaal neither advocates nor endorses the use of the presented techniques to analyze this type of auction.

H. Houba $(\bowtie) \cdot$ D. van der Laan

Department of Econometrics, VU University Amsterdam, Tinbergen Institute, De Boelelaan 1105, 1081 HV Amsterdam, Netherlands

e-mail: hhouba@feweb.vu.nl

D. van der Laan

e-mail: dalaan@feweb.vu.nl

D. Veldhuizen

SNS Reaal, Group Risk Management, Croeselaan 1, 3503 RK Utrecht, The Netherlands

e-mail: Dirk.Veldhuizen@snsreaal.nl 


\section{Introduction}

During the last decade, the lowest-unique bid auction appeared on several radio and TV shows and on the internet. In such auctions, the winning bid is the lowest bid among the unique bids made, being those bids that are made only once. The items auctioned are typically fancy mass-produced consumer electronics, cars, or monetary prizes. Bids are typically made by sending a costly SMS-call with the bid expressed in cents. Most of the time, the winning bid pays almost nothing. In these auctions, potential bidders face strategic uncertainty about how many other bidders actually submit bids and the spread of bids submitted by others. Other important issues are the profitability of submitting a bid, the actual decision which bid to submit and to taken into account the option of non-participating.

Lowest-unique-bid auctions are a new auction format with discrete bids that is not captured by mainstream auction theory, as e.g., surveyed in Krishna (2002). Thus far, the sealed-bid version of this auction format is only studied in Östling et al. (2007), Rapoport et al. (2007) and Eichberger and Vinogradov (2008). The first two references study a single costless bid per bidder, whereas the last reference allows bidders to submit multiple costly bids. The issue of endogenous entry is recognized in Eichberger and Vinogradov (2008), while Rapoport et al. (2007) consider implementing costly entry as being too complicated in laboratory experiments. Östling et al. (2007) model the strategic uncertainty about entry as a large Poisson game, as proposed in Myerson $(1998,2000)$. This approach, however, considers entry as a black box and the primitive of the Poisson process is not related to strategic deliberations by potential bidders that depend upon the entry cost and the value of the item.

Despite the simplicity of the auction format, all references recognize that the lowest-unique sealed-bid auction is hard to analyze. In these references, the emphasis is to test data from either laboratory or field experiments, and the theory is developed up to the point where numerical computations can be performed, which is needed for the statistical tests. Östling et al. (2007) do provide a characterization result for this auction format, but only under unrealistic additional assumptions for the special case of large Poisson games, such as costless bids and the winner does not have to pay his winning bid. Then, uniqueness of a symmetric Nash equilibrium (NE) is obtained with a full support over feasible bids, bidding probabilities that decrease as bids become larger, and convergence to the uniform distribution over bids as the number of bidders goes to infinity. Our article is theoretically motivated to better understand this auction format under realistic assumptions regarding costly entry and the winner pays his winning bid. And, as we will show, realistic assumptions imply different results.

In this article, we study lowest-unique sealed-bid auctions in which bidders submit a single costly bid, being a discrete number, and the cost of this bid is confected when submitting it. Endogenous entry is modeled by allowing the costless bid "do not participate". All bidders have a common value for the auctioned item, which is similar as in the references mentioned. This simple model offers a natural framework 
to simultaneously analyze strategic uncertainty about costly entry and bid behavior. As in the references, we only study symmetric NEs. ${ }^{1}$

Any symmetric NE in our model is in mixed strategies, but not with a full support on the set of numerical bids as in Östling et al. (2007). Instead, the support contains a consecutive subset of numerical bids that includes the lowest bid. Moreover, higher bids within the support are played with a lower probability, but higher bids do have a larger probability of winning. ${ }^{2}$ This reflects the intuition that one should try to bid low, but if everyone would do so, it is better to avoid the overcrowded lower bids and consider somewhat higher bids. If the expected NE gains are positive, then all bidders participate for sure. A positive probability of non-participating, however, implies that these expected gains are zero.

Although both the simple two-bidder case and the lowest-bid auction allow for a straightforward characterization, we show that the characterization of the symmetric NE involves solving equations, in which polynomials of large order need to be solved and that such characterization cannot be achieved for arbitrary large number of bidders. Under positive expected NE gains and a technical condition, however, we characterize the symmetric NE with the lowest expected NE gains as the minimum of a mathematical program. The interpretation of this symmetric NE is that it is the maximin value within the class of symmetric strategies. Therefore, a bidder's bidding strategy also minimizes what this bidder "gives away" in expected NE gains to the other bidders. Positive expected NE gains include the case of zero bidding cost, making the unmodeled entry in Östling et al. (2007) and Rapoport et al. (2007) trivial.

We also perform comparative statics and find sufficient conditions stating that if either the number of bidders or the cost of making a bid becomes too large, then the probability of non-participating becomes positive and the expected gains in any symmetric NE are zero. And, as the number of bidders goes to infinity, we have convergence to a limit distribution that is uniform over numerical bids, albeit a trivial sort of uniform distribution with limit probabilities on numerical bids equal to zero and the individual probability of non-participating equal to one, which is the opposite of the convergence result in Östling et al. (2007) mentioned above. For a sufficiently large value of the item or low enough bidding cost, there is always a positive expected gain and, hence, full participation. This implies that a bidding cost of zero is a special case and not the general one. If the value would go to infinity, the support of numerical bids becomes unbounded. We also derive a partial invariance of symmetric NE bidding strategies with respect to bidding cost: If, for bidding cost equal to 0 , a bidding strategy is a NE strategy with positive expected gains of say 11.3, then this NE strategy remains

\footnotetext{
${ }^{1}$ Lowest-unique sealed-bid auctions admit a staggering amount of asymmetric NEs. Even for a fixed number of bidders, the number of NEs rapidly grows under an increasing value of the auctioned item. Houba et al. (2008) report the following for three bidders and unit cost: Twenty-one asymmetric NEs and one symmetric NE when the value of the item is 5 . For a value of 15 , there are 42 asymmetric NEs and one symmetric. This suggests that the prospects for a full characterization of NEs are too slim to embark on such a project.

2 Similar results are derived in Raviv and Virag (2008) for a related auction format, in which bids are restricted to be below 5 or $10 \%$ of the value of the item and the highest-unique sealed bid wins. Also, Eichberger and Vinogradov (2008) report that bidders randomize over consecutive sets of numerical bids that include the lowest bid.
} 
a NE strategy under all bidding costs 1 up to 11 . For larger bidding costs, there exists a NE with expected gains equal to zero. Some implications for experimental studies are discussed in the concluding remarks.

We also observe that the two bidders' auction is equivalent to the Hawk-Dove game and, hence, the symmetric NE is unique and evolutionary stable. Such stability reflects that learning how to play best responses in this auction format leads to the symmetric NE. Such dynamics certainly will play a role in reality, where these auctions are held repeatedly. For that reason, we also numerically investigate asymptotic stable equilibria of the replicator dynamics as a proxy for evolutionary stable strategies, see Bukowski and Miekisz (2004). ${ }^{3}$ For the parameter values run, we observe convergence to the symmetric NE with the lowest expected gains. Simulation of replicator dynamics is slow and in the case of positive expected gains running the mathematical program is faster in obtaining numerical solutions. For that reason, we regard both types of numerical analyses as complementary methods in computing the symmetric NE with lowest expected gains.

This article is organized as follows. We first introduce our model in Sect. 2. In Sect. 3, we discuss motivating examples that identify the main issues of concern. All theoretical results are derived in Sects. 4, 5, and 6, where Sect. 5 is devoted to the mathematical program. A numerical analysis of the replicator dynamics and the mathematical program are reported in Sect. 7. This article concludes with some remarks.

\section{The lowest-unique sealed-bid auction}

In a lowest-unique sealed-bid auction with endogenous entry, each bidder $(b=$ $1,2, \ldots, n+1, n+1 \geq 3$ ) either stays out (makes the non-participating bid $\beta^{b}=N$ ) or enters by submitting a costly integer bid $\left(\beta^{b} \in \mathbb{N}_{0} \equiv\{0\} \cup \mathbb{N}\right)$ for an item. All bidders value the item as $M \in \mathbb{N}$ and submitting an integer bid costs $c \in \mathbb{N}$, where $c<M$ is the non-trivial case. Cost $c$ of submitting a bid are forfeited immediately. Consider the (possibly empty) set of all bids that are unique among the submitted bids, then the winning bid is the lowest bid in this set. The winner of the auction receives the item and also pays his own bid. Note that the existence of a winning bid is not guaranteed, as for example, $n+1$ identical bids demonstrate. In the absence of a winner, the item remains with the auctioneer.

An outcome of the auction is denoted $\left(\beta^{b}, \beta^{-b}\right) \in B^{n+1} \equiv \prod_{i=1}^{n+1} B$, where $\beta^{-b} \in$ $\prod_{i=1}^{n} B$ is a vector that consists of bidder $b$ 's opponents bids. Every outcome specifies a possibly empty set of unique bids and, if non-empty, the winner is the bidder who has the lowest (unique) bid in this set. Given $\left(\beta^{b}, \beta^{-b}\right) \in B^{n+1}, I\left(\beta^{b}, \beta^{-b}\right) \in$ $\{\{1\}, \ldots,\{n+1\}\} \cup \emptyset$ denotes the identity of the winner, where $I\left(\beta^{b}, \beta^{-b}\right)=\varnothing$ indicates in the absence of a winner. Under complete information, the payoff to bidder $b$ is given by

\footnotetext{
3 Our attempts to characterize a unique evolutionary stable equilibrium failed, but as a side result did yield the mathematical program mentioned above. Therefore, we resort to a numerical analysis.
} 


$$
u_{b}\left(\beta^{b}, \beta^{-b}\right)= \begin{cases}0, & \text { if } \beta^{b}=N \\ M-c-\beta^{b}, & \text { if } I\left(\beta^{b}, \beta^{-b}\right)=b \\ -c, & \text { otherwise. }\end{cases}
$$

Since the winner pays his submitted bid, bidding above $M-c$ is dominated by non-participating. Hence, it is without loss of generality to assume that $B=\{N\} \cup$ $\{0,1, \ldots, M-c\}$ is the set of feasible bids. For the subset of integer bids, we write $B^{0}=B \cap \mathbb{N}_{0}$. A mixed strategy $x=\left(x_{N}, x_{0}, x_{1}, \ldots, x_{M-c}\right)$. The simplex of mixed strategies on $B$ is denoted as $\Delta=\left\{x \in \mathbb{R}_{+}^{M-c+2} \mid \sum_{i \in B} x_{i}=1\right\}$. We write $x^{b} \in \Delta$ for bidder $b$ 's mixed strategy and $x^{-b} \in \prod_{i=1}^{n} \Delta$ as the mixed strategies of bidder $b$ 's opponents. In order to summarize, the lowest-unique sealed-bid auction is a symmetric normal-form game with finite pure-strategy sets.

A preliminary result identifies the minimax value as the payoff from non-participating and establishes existence of symmetric NEs.

Proposition 1 Bidder b's minimax value is 0 and all expected NE payoffs are nonnegative. There exists a symmetric mixed-strategy NE and all such NEs feature randomization.

Proof Bid $\beta^{b}=N$ secures bidder $b$ a payoff of 0 . All other bidders can hold $b$ down to 0 by coordination on $\beta^{-b}$ such that a single bidder $k \neq b$ bids $\beta^{k}=0$ and all others bid $\beta^{l}=N, l \neq b, k$. Hence, 0 is $b$ 's minimax value (and these minimax strategies are NE). For every NE, NE payoffs weakly dominate minimax payoffs, which are all 0. Next, the conditions of Theorem 1 in Becker and Damianov (2006) hold, which proves the existence of a symmetric NE. Finally, any symmetric NE must involve the randomization over at least two bids in $B$. Suppose not, then for some $\alpha \in B$ the pure strategy profile $\left(\beta^{b}, \beta^{-b}\right)=(\alpha, \ldots, \alpha)$ forms a symmetric NE. In such NE $I\left(\beta^{b}, \beta^{-b}\right)=\emptyset$ and $b$ 's payoff is at most 0 , if not negative. If $\alpha \neq 0$, then $\beta^{b}=0$ is bidder $b$ 's optimal deviation with $I\left(0, \beta^{-b}\right)=b$ for sure and $u_{b}\left(0, \beta^{-b}\right)=M-c>0$. Similar, for $\alpha=0, \beta^{b}=1$ is the optimal deviation $I\left(1, \beta^{-b}\right)=b$ for sure and $u_{b}\left(1, \beta^{-b}\right)=M-c-1>-c$. Hence, there does not exist any pure symmetric $\mathrm{NE}$.

The model setup captures the reduced form of a two-stage game with unobservable entry, in which potential bidders first decide whether to participate $(P)$ and, if so, decide their sealed bid. In this extensive form, $b$ 's behavior strategy is either of the form $\left(P, \beta^{b}\right)$, or $\left(N, \beta^{b}\right), \beta^{b} \in \mathbb{N}_{0}$. Since strategies $\left(N, \beta^{b}\right)$ and $\left(N, \tilde{\beta}^{b}\right)$ are payoff equivalent, we may simply write $\left(N, \beta^{b}\right)$ as $N$ and $\left(P, \beta^{b}\right)$ as $\beta^{b}$. The model also captures auctions that specify minimum bids $\beta^{\min } \in \mathbb{N}, \beta^{\text {min }}<M$, because such auctions are strategically equivalent to auctions with $M-\beta^{\min }$ and integer bids in $\mathbb{N}_{0}$. The common value $M$ captures the fact that many real lowest-unique bid auctions have some mass-produced consumer good as the auctioned item and such item is typically on sale through (internet) shops, hence, available against a uniform market price.

Under symmetric mixed strategies $x$ (which allows dropping superscript $b$ ), the random number of participating bidders follows a Binomial distribution Bin $(n+1$, $\left.1-x_{N}\right)$, where $x_{N}$ is the probability of non-participating. Therefore, the expected number of bidders is $(n+1)\left(1-x_{N}\right)$ with a standard deviation of $\sqrt{(n+1) x_{N}\left(1-x_{N}\right)}$. 
Obviously, if $x_{N}=0$, then all $n+1$ bidders enter for sure. Similar, $x_{N}=1$ implies no bidder enters for sure. These last properties are different when compared to the large variance in large Poisson games, see Myerson (1998, 2000), where the variance can only be close to zero if and only if the expected number of participants is close to zero. ${ }^{4}$

\section{Motivation}

The motivation for our analysis is to better understand symmetric NEs in this type of auction and how these resolve the strategic uncertainty. In this section, we argue that the strategic uncertainty and also the equilibrium analysis, is much more complicated than in other auction formats, such as the lowest-bid auction.

The case $n+1=2$ is trivial. In order to see this, $n+1$ imposes the bi-matrix game:

\begin{tabular}{llllll}
\hline & $N$ & 0 & 1 & $\cdots$ & $M-c$ \\
\hline$N$ & 0,0 & $0, M-c$ & $0, M-c-1$ & $\cdots$ & 0,0 \\
0 & $M-c, 0$ & $-c,-c$ & $M-c,-c$ & $\cdots$ & $M-c,-c$ \\
1 & $M-c-1,0$ & $-c, M-c$ & $-c,-c$ & $\cdots$ & $M-c-1,-c$ \\
$\vdots$ & $\vdots$ & $\vdots$ & $\vdots$ & $\ddots$ & $\vdots$ \\
$M-c$ & 0,0 & $-c, M-c$ & $-c, M-c-1$ & $\cdots$ & $-c,-c$ \\
\hline
\end{tabular}

It admits an inefficient symmetric mixed-strategy NE in which bidder $b=1,2$ submits the lowest bid 0 with probability $x_{0}^{b}=1-\frac{c}{M}$ and does not participate with probability $x_{N}^{b}=\frac{c}{M}$. In the symmetric NE, the probability that at least one bidder participates is $1-\left(\frac{c}{M}\right)^{2}$. This implies that the expected number of bidders increases in $M$ and decreases in $c$. None of the bidders expects to make a positive payoff from participating, because all bids in the support are payoff equivalent to the payoff of non-participating. The probability that nobody wins the auction is $\left(\frac{c}{M}\right)^{2}+\left(1-\frac{c}{M}\right)^{2}$. In the case of costless bidding, $\beta^{b}=0$ and no winner for sure.

Several remarks are in place. First, consider the asymmetric modification in which bidder 1 values the item as $M+\varepsilon$, for sufficiently small $\varepsilon>0$. Then, $x_{0}^{1}=\frac{c}{M}>$ $\frac{c}{M+\varepsilon}=x_{0}^{2}$ implies that the Pareto inefficient allocation of the item to bidder 2 has positive probability. Hence, lowest-unique sealed-bid auctions do not allocate items efficiently, and we consider further investigation of (in)efficiency issues as uninteresting. Second, from the perspective of the auctioneer, the bidding behavior in the asymmetric NEs is identical to that of a bidding ring, which does not adequately describe internet auctions where bidders are anonymous. We therefore disregard asymmetric NEs and refer to Houba et al. (2008) for a discussion of this subject.

The two-bidder auction is similar in interpretation to the Hawk-Dove (HD) game, where two animals contest a single prize under cost of fighting, see e.g., Weibull (1995), for a survey of evolutionary game theory. Both games have a symmetric mixed NE. Therefore, lowest-unique sealed-bid auctions can be seen as generalized HD games

\footnotetext{
${ }^{4}$ If the number of players $X$ is Poisson distributed with parameter $N$, then $E(X)=V A R(X)=N$. Therefore, arbitrary $E(X)$ and $V A R(X) \neq N$ cannot be studied in large Poisson games.
} 
Table 1 Symmetric NEs for several values of $M, n+1=3$, and $c=1$

\begin{tabular}{llllllll}
\hline$M$ & $x_{N}$ & $x_{0}$ & $x_{1}$ & $x_{2}$ & $x_{3}$ & NE payoff & Sum payoffs \\
\hline 3 & 0.5669 & 0.4227 & 0.0105 & & & 0.0000 & 0.0000 \\
4 & 0.2887 & 0.5000 & 0.2113 & & & 0.0000 & 0.0000 \\
5 & & 0.5191 & 0.3407 & 0.1403 & & 0.1565 & 0.4695 \\
6 & & 0.5093 & 0.3189 & 0.1717 & & 0.4447 & 1.3341 \\
10 & & 0.4904 & 0.2902 & 0.2154 & 0.0040 & 1.5969 & 4.7907 \\
15 & & 0.4801 & 0.2769 & 0.1750 & 0.0680 & 3.0544 & 9.1632 \\
25 & & 0.4713 & 0.2659 & 0.1577 & 0.1051 & 5.9886 & 17.9668 \\
\hline
\end{tabular}

with a single prize, multiple contesters and a richer strategy structure for each contester. Finally, after restricting the auction's strategy space to $\{N, 0\}$, both two-player models can be reduced to the same generic form with negative diagonal elements. Hence, the mixed NE is the unique evolutionary stable equilibrium (ESS) in the restricted two-bidder auction.

Next, consider the lowest-unique sealed-bid auction with $n+1=3$ and $c=1$. For several values of $M$, we numerically compute symmetric NEs by implementing the auction in the software tool Gambit, see e.g., McKelvey et al. (2006). All numerical computations yield a unique symmetric NE. It is in mixed strategies that are not fully mixed over $B$. Comparative statics with respect to the value of $M$ in Gambit, as reported in Table 1, shows that $M=5$ is the lowest $M$ for which all bidders participate for sure because the expected NE payoffs are positive. For smaller $M$, non-participating has positive probability in the symmetric NE and the bidders' expected NE payoffs are zero. The support of monetary bids consists of decreasing probabilities on consecutive numbers and its size increases in $M$. In Gambit, the symmetric NEs of Table 1 can also be computed as the quantal response equilibrium, see McKelvey and Palfrey (1995). Although this equilibrium concept suggests an evolutionary underpinning, examples exist for which such equilibrium fails to be evolutionary stable. Therefore, evolutionary stability of either symmetric NEs or the unique quantal response equilibrium is an open issue.

A somewhat different issue is which feature of the unique-lowest sealed-bid auction complicates the equilibrium analysis. Is this related to making the lowest or the unique bid? To answer this question, we characterize the NEs of the lowest (sealed)bid auction with endogenous entry, where ties among multiple lowest bids are broken randomly. In this auction, bidder $b$ 's payoff is given by

$\hat{u}_{b}\left(\beta^{b}, \beta^{-b}\right)= \begin{cases}0, & \text { if } \beta^{b}=N, \\ \frac{M-\beta^{b}}{k}-c, & \text { if } \beta^{b} \in \min \left\{\beta^{b}, \beta^{-b}\right\} \text { and } k \text { is number of lowest bids } \\ -c, & \text { otherwise. }\end{cases}$

The following proposition characterizes the symmetric NE.

Proposition 2 For the lowest-bid auction, we have that: 
(1) If $c<\frac{M}{n+1}$, then the unique NE is given by $x_{0}=1$ and expected NE payoffs of $\frac{M}{n+1}-c$.

(2) If $\frac{M}{n+1}<c<M$, then (i) the symmetric $N E$ is given by the unique $x_{0} \in(0,1)$ :

$$
\frac{1}{n+1} \cdot \frac{1}{x_{0}}\left(1-\left(1-x_{0}\right)^{n+1}\right)=\frac{c}{M},
$$

$x_{N}=1-x_{0}$ and expected NE payoffs of 0; (ii) other asymmetric NEs exist.

Proof (1) Consider mixed strategies $x^{-b}$ and integer bid $\beta^{b}=0$. Whatever realization $\beta^{-b}, 0 \leq \min \left\{0, \beta^{-b}\right\}$ and $b$ participates for sure in the lottery for the item with positive expected payoff. In fact, $\beta^{b}=0$ is the unique best response to any realization $\beta^{-b}$ independent of the probability distribution $x^{-b}$ imposes over $\beta^{-b}$.

(2) In any symmetric NE $x$, the support of $x$ is $\{N, 0\}$, because from 1 . it follows that $\beta^{b}=0$ dominates $\tilde{\beta}^{b} \geq 1$. Given $x_{0} \in(0,1)$, the expected number of other bidders who bid 0 , denoted as $k$, follows the Binomial distribution $\operatorname{Bin}\left(n, x_{0}\right)$. Therefore, the expected gain of bidding $\beta^{b}=0$ against $x^{-\beta}$ is proportional to the probability $f\left(x_{0}\right)$ of winning that is equal too

$$
\begin{aligned}
f\left(x_{0}\right) & =\sum_{k=0}^{n}\left(\begin{array}{l}
n \\
k
\end{array}\right) x_{0}^{k}\left(1-x_{0}\right)^{n-k} \cdot \frac{1}{k+1} \\
& =\frac{1}{n+1} \cdot \frac{1}{x_{0}} \sum_{k=0}^{n}\left(\begin{array}{l}
n+1 \\
k+1
\end{array}\right) x_{0}^{k+1}\left(1-x_{0}\right)^{n+1-(k+1)} \\
& =\frac{1}{n+1} \cdot \frac{1}{x_{0}}\left(1-\left(1-x_{0}\right)^{n+1}\right)
\end{aligned}
$$

In any mixed NE, pure strategies in the support are payoff equivalent. Therefore, $\beta^{b}=0$ is payoff equivalent to $\beta^{b}=N$ and, hence, $M \cdot f\left(x_{0}\right)-c=0$, which implies (1). Since $f\left(x_{0}\right)$ is monotonically decreasing, $\lim _{x_{0} \rightarrow 0} f\left(x_{0}\right)=1$ and $f(1)=\frac{1}{n+1}$, there exists a unique $x_{0}$ such that $f\left(x_{0}\right)=\frac{c}{M}$.

We provide an asymmetric NE in pure strategies: $\ell \in \mathbb{N}$ is the maximum number such that $\frac{M}{\ell}>c$. Then, $1 \leq \ell \leq n+1$ exists. Consider the pure strategies, Bidder $b=1, \ldots, \ell$ submits $\beta^{b}=0$ and bidder $b=\ell+1, \ldots, n+1$ submits $\beta^{b}=N$. These asymmetric pure strategies form a NE. Also, any permutation of bidders' roles is also NE.

The proof of this proposition illustrates that the lowest-bid auction can be considered as an exercise in game theory. In essence, the lowest-bid auction boils down to a lottery with an endogenous winning probability. Condition (1) admits $x_{0}^{b}=2\left(1-\frac{c}{M}\right) \in(0,1)$ for $n+1=2$ and, for larger $n+1$, it can be numerically solved by mathematical software packages. The comparative statics in the case 2 are straightforward, $x_{0}$ increases in $M$ and decreases in $c$ and $n+1$. Furthermore, $x_{0}$ is equal to 1 for large $M \geq \frac{c}{2}$. And, $x_{0}$ goes to 0 as $\frac{c}{M}$ goes to 1 . The cause of the multiplicity of NEs can be attributed to 
costly endogenous entry and its intuition is similar to the multiplicity of equilibria in symmetric market entry models. As our analysis will make clear, symmetric NEs in the lowest-unique sealed-bid auction are non-trivial, and it can be attributed to requiring that the winning bid should be a unique bid. This fact is also illustrated by the NE supports in Table 1 that consist of several numerical bids, and its length is increasing in $M$.

In order to summarize, lowest-unique sealed-bid auctions probably admit a unique symmetric NE with randomization over several numerical bids. The strategic uncertainty is much more complex and non-trivial than in other auction formats, such as the lowest-bid auction. The similarity with the HD game suggests that the symmetric NE might also be an ESS in the general case. Our analysis, therefore, concentrates on symmetric NEs and $n+1 \geq 3$.

\section{Symmetric Nash equilibrium}

In this section, we establish that several properties observed in Table 1 hold in general. For notational convenience, we take the perspective of bidder 1 meaning $x \in \Delta$ is a symmetric NE if and only if $x^{1}=x$ is a best response against $x^{-1}=(x, \ldots, x)$. The non-empty set of symmetric NE is denoted as $\Delta^{N E}$. The support of $x$ is denoted as $S(x) \subseteq B$ and for its subset of monetary bids, we write $S^{0}(x) \subseteq B^{0}$.

Theorem 3 Let $x \in \Delta^{N E}$. Then, the numerical support $S^{0}(x)$ is a set of consecutive numbers containing the lowest bid of 0 . The probability $x_{i}$ is strictly monotone decreasing in $i \in S^{0}(x)$ and bid $i$ 's probability of winning is strictly monotone increasing in $i \in S^{0}(x)$.

Before proceeding to the formal proof, we first discuss this result. A support of consecutive numbers means that there are no gaps of bids that will never be chosen. The rationale is that any bid in such gap would allow to undercut all realizations of NE bids above the gap by having a unique bid for sure. And, if such bid would prevail as the winning bid, the bid is also less expensive than any bid above the gap. Since the probability that such undercutting bid is winning is positive, and has at least a probability of winning equal to those of the higher NE bids it undercuts, it therefore does strictly better than the NE bids above this gap. This argument also implies that the lowest bid cannot be part of any gap and, therefore, must be included in the support.

Symmetric NEs underpin the complicated deliberations of bidding in the lowestunique sealed-bid auction. On the one hand, the rules imply that bidding the lowest bid or close to it should be considered, because otherwise someone else might undercut. On the other hand, if everybody bids very low, the lower bids become overcrowded and it is better to contemplate a somewhat higher bid. Since such bids have more potential lower bids that might be winning, these bids are less attractive and, therefore, such bids are made with lower probabilities. Decreasing probabilities over bids, however, also imply that lower bids have higher probabilities of being non-unique and, in any symmetric NE, have lower probabilities of winning. Higher bids in the support have higher probabilities of winning, since this is needed to offset the extra costs of higher bids. Mixed symmetric NE strategies delicately trade off both effects. In order to sum- 
marize, mixed symmetric NEs predict that lower bids are more likely to be observed and that higher bids win relatively more often compared to lower bids.

We now turn our attention to the proof of Theorem 3. From bidder 1's perspective, define $F_{i} \subseteq B^{n}, i \in B^{0}$, as the set of partial outcomes $\beta^{-1}=\left(\beta^{2}, \ldots, \beta^{n+1}\right)$ such that $I\left(i, \beta^{-1}\right)=1$, meaning $i$ is a winning bid for bidder 1 given $\beta^{-1}$. This implies that, if $k_{j}\left(\beta^{-1}\right)$ is the number of bids equal to $j \in B^{0}$ in $\beta^{-1}$, then $\beta^{-1} \in F_{i}$ if and only if $k_{j}\left(\beta^{-1}\right) \neq 1$ for all $j=1, \ldots, i-1$ and $k_{i}\left(\beta^{-1}\right)=0$.

Given the mixed strategy $x \in \Delta$, let $f_{i}(x)$ be the probability that bidder 1 can win by making the bid $i \in B^{0}$ given that all other bidders use the mixed strategy $x$, i.e., $x^{-1}=(x, \ldots, x)$. Then, we have for every $i \in B^{0}$ that

$$
f_{i}(x)=\sum_{s \in F_{i}} \operatorname{Pr}\left(\beta^{-1}=s\right)=\sum_{s \in F_{i}} x_{s^{2}} \cdots x_{s^{n+1}} \equiv \sum_{s \in F_{i}} \operatorname{Pr}(s)
$$

where $s=\left(s^{2}, \ldots, s^{n+1}\right) \in B^{n}$. Obviously, $f_{i}(x)$ is continuous in $x$. Bidder 1's expected utility of bid $i \in B^{0}$ against $x^{-1}$ is denoted as $u(i, x)=(M-i) f_{i}(x)-c$. The expected symmetric NE utility is denoted as $u(x, x)=\sum_{i \in S^{0}(x)} x_{i} u(i, x)$ and this function is continuous in both $x^{1}=x$ and $x^{-1}=(x, \ldots, x)$.

The first lemma follows directly from the definition of the NE concept, all pure strategies in the support of a mixed NE are optimal pure strategies and payoff equivalent.

Lemma 4 Let $x \in \Delta^{N E}$. For all $i \in S^{0}(x)$ and $j \in B^{0},(M-i) f_{i}(x) \geq(M-j) f_{j}(x)$ and equality holds if also $j \in S^{0}(x)$. Moreover, $N \in S(x)$ implies $u(x, x)=0$.

Lemma 4 implies the next result, which proves Theorem 3.

Lemma 5 If $x \in \Delta^{N E}$, then $x_{i}$ and $f_{i}(x)$ are strictly monotone decreasing, respectively, increasing in $i \in S^{0}(x)$. The expected payoff $u(i, x)$ is non-increasing in $i \in B^{0}$, i.e., $u(i, x) \geq u(i+1, x)$ for $i \in B^{0}$. Moreover, there exists a $\bar{\beta} \in B^{0}$ such that $S^{0}(x)=\{0, \ldots, \bar{\beta}\}$.

Proof Suppose that for $i, i+1 \in S^{0}(x)$, we would have $x_{i+1} \geq x_{i}$. Let $\beta^{-1} \in F_{i+1}$. Then for $j=0, \ldots, i$, we have that $k_{j}\left(\beta^{-1}\right) \neq 1$ and $k_{i+1}\left(\beta^{-1}\right)=0$. From $\beta^{1}$, we construct bids $\hat{\beta}^{-1}=\hat{\beta}^{-1}\left(\beta^{-1}\right)$ such that $\hat{\beta}^{-1} \in F_{i}$ by moving all bids $\beta^{b}=i$, $b=2, \ldots, n+1$, to $\hat{\beta}^{b}=i+1$. Formally, let $\hat{\beta}^{-1}=\hat{\beta}^{-1}\left(\beta^{-1}\right)=\left(\hat{\beta}^{2}, \ldots, \hat{\beta}^{n+1}\right)$, where for $b=1, \ldots, n$ we take $\hat{\beta}^{b}=i+1$ if $\beta^{b}=i$ and $\hat{\beta}=\beta^{b}$ otherwise. Then, for $\hat{\beta}^{-1}$ we have that $k_{j}\left(\beta^{-1}\right) \neq 1$ for $j=0,1, \ldots, i-1$ and $k_{i}\left(\beta^{-1}\right)=0$. Hence, $\hat{\beta}^{-1} \in F_{i}$. Moreover, from $x_{i+1} \geq x_{i}$ it follows that $\operatorname{Pr}\left(\hat{\beta}^{-1}\left(\beta^{-1}\right)\right) \geq \operatorname{Pr}\left(\beta^{-1}\right)$, because all $x_{s}=x_{i}$ become $x_{s} b=x_{i+1}$ in (2). However, then we have

$$
f_{i+1}(x)=\sum_{\beta^{-1} \in F_{i+1}} \operatorname{Pr}\left(\beta^{-1}\right) \leq \sum_{\hat{\beta}^{-1}\left(\beta^{-1}\right) ; \beta^{-1} \in F_{i+1}} \operatorname{Pr}\left(\hat{\beta}^{-1}\left(\beta^{-1}\right)\right) \leq \sum_{s \in F_{i}} \operatorname{Pr}(s)=f_{i}(x)
$$

and this implies $(M-(i+1)) f_{i+1}(x)<(M-i) f_{i}(x)$ with $i, i+1 \in S^{0}(x)$. This contradicts Lemma 4. Thus, if $i, i+1 \in S^{0}$ then $x_{i+1}<x_{i}$. Then, by Lemma 4, for 
any $i, i+1 \in S^{0}(x)$, we have that $f_{i}(x)=\frac{M-i-1}{M-i} f_{i+1}(x)<f_{i+1}(x)$. The expected payoff $u(i, x) \geq u(i+1, x)$ for all $i \in S^{0}(x)$ by the definition of a NE. For $i \notin S^{0}(x)$, if $i+1$ would be an unique-lowest bid, then so is $i$. Hence, $f_{i}(x) \geq f_{i+1}(x)$ and $u(i, x)>u(i+1, x)$. Finally, by Proposition $1, \bar{\beta} \in B^{0}$ exists. For $i \leq \bar{\beta}: x_{i}>x_{\bar{\beta}}>0$ implies $i \in S^{0}(x)$.

At this point, it is worthwhile to address the complex nature of characterizing symmetric NE in the lowest-unique sealed-bid auction. In order to facilitate this discussion, we first introduce formulas for the winning probability $f_{i}(x)$ of bid $i \in B^{0}$ defined by (2) and state some elementary properties needed below. For $i \in B^{0}$, we can express $f_{i}(x)$ as:

$$
\begin{aligned}
f_{0}(x) & =\left(1-x_{0}\right)^{n}, \\
f_{1}(x) & =\sum_{k_{0} \neq 1}^{n}\left(\begin{array}{c}
n \\
k_{0}
\end{array}\right) x_{0}^{k_{0}}\left(1-x_{0}-x_{1}\right)^{n-k_{0}}, \\
f_{i}(x) & =\sum_{\substack{k_{0}+k_{1}+\ldots+k_{i-1}+k_{i}=n \\
k_{j} \neq 1, j=0,1, \ldots, i-1}}^{n}\left(\begin{array}{c}
n \\
k_{0}, k_{1}, \ldots, k_{i-1}, k_{i}
\end{array}\right) \cdot x_{0}^{k_{0}} \cdot x_{1}^{k_{1}} \cdots x_{i-1}^{k_{i-1}} \cdot r_{i}^{k_{i}}(x),
\end{aligned}
$$

where $r_{i}(x)=1-x_{0}-\cdots-x_{i}$ is the complementary probability that an individual bid does not belong to the set $\{0,1, \ldots, i\}$ and the multinomial coefficient is defined as

$$
\left(\begin{array}{c}
n \\
k_{0}, k_{1}, \cdots, k_{i-1}, k_{i}
\end{array}\right)=\frac{n !}{k_{0} ! \cdot k_{1} ! \cdots k_{i-1} ! \cdot k_{i} !} .
$$

In a symmetric $\mathrm{NE}$, all $n+1$ players have the same probability of winning with the lowest-unique bid and, moreover, there is at most one winner. This implies the following result.

Lemma 6 Let $x \in \Delta^{N E}$. Then, for all $i \in B^{0}: f_{i}(x) \leq \frac{1}{n+1}$.

From this lemma, $f_{0}(x)=\left(1-x_{0}\right)^{n}$ and $u(x, x)=u(0, x) \geq 0$, we can derive

$$
0<1-\left(\frac{1}{n+1}\right)^{1 / n} \leq x_{0}=1-\left(\frac{c+u(x, x)}{M}\right)^{\frac{1}{n}} \leq 1-\left(\frac{c}{M}\right)^{\frac{1}{n}}<1 .
$$

The summation of large powers in the probabilities $f_{i}(x)$ for $i \geq 1$ obstructs any approach to obtain similar closed-form solutions for $x_{1}, x_{2}, \ldots$, except for the quadratic case $n+1=3$ (which we omit). This shows that the lowest-unique sealed-bid auction is fundamentally different than the lowest-bid auction in Proposition 2.

In principle, the unknown $u(x, x)$ and the probabilities $x \in \Delta^{N E}$ can be numerically computed by applying the bisection method to update $u(x, x) \in\left[0, \frac{M}{n+1}-c\right]$. Rapoport et al. (2007) claim that this guarantees convergence to the true value of 
$u(x, x)$ under $c=0$, whereas Östling et al. (2007) claim numerical solving is explosive in the number of bidders and is better avoided when $n+1 \geq 9$. In Sect. 5, we develop an alternative approach, in which we identify a particular $x \in \Delta^{N E}$ as the solution of a mathematical program that also allows an economic interpretation.

We conclude this section by deriving several small results from (3) needed for later purposes. The first result can be verified from (3) and is stated without a proof.

Lemma 7 For $i \in B^{0}, f_{i}(x)$ is continuous on $\Delta$ and symmetric in the variables $x_{0}, x_{1}, \ldots, x_{i-1}$.

The next result states some properties of the following modification of $f_{i}$ in which the variable $r_{i} \in \mathbb{R}_{+}$replaces the function $r_{i}(x)$. We call this function the modified function $f_{i}$. These properties can also be verified from (3).

Lemma 8 The modified function $f_{i}(x)$ is non-decreasing in the variables $x_{0}, x_{1}, \ldots$, $x_{i-1}, r_{i}$ and, increasing if all $x_{0}, x_{1}, \ldots, x_{i-1}, r_{i}$ are positive.

\section{A mathematical program for symmetric NEs}

In the previous section, we have argued that the formulas for the winning probabilities in (3) do not allow for a closed-form solution. Instead, we show that the most competitive symmetric NE is the solution of a mathematical program that allows for an economic interpretation. The mathematical program is given by

$$
\begin{aligned}
& \min _{z \geq 0 ; x \geq 0} z \\
& \text { s.t. } \\
& u(i, x) \quad \leq z, i \in B^{0}, \\
& \sum_{i \in B^{0}} x_{i} \leq 1 .
\end{aligned}
$$

Since all $u(i, x)$ are independent of $x_{N}$, we have implicitly defined this probability as the slack in the last constraint.

The following results are rather straightforward. Let $x \in \Delta^{N E}$ and $z=u(x, x) \geq 0$, then the standard properties of the NE concept imply that $(z, x)$ is a feasible solution of (5). This fact implies a non-empty set of feasible solutions and that the optimal $z \geq 0$ is bounded from above by $\min _{x \in \Delta^{N E}} u(x, x)$. Furthermore, the subset of $x \geq 0$ such that $\sum_{i \in B^{0}} x_{i} \leq 1$ is a non-empty, compact, convex set and, by (3), $u(i, x), i \in B^{0}$, is a continuous function in $x$. This suffices for program (5) to admit an optimal solution.

The main result states two conditions under which the optimal solution $(z, x)$ of (5) implies $x \in \Delta^{N E}$ : the expected utility $z$ in the optimal solution has to be positive and a technical condition on the probabilities of winning. The technical condition is associated with a particular redistribution of probability mass over two consecutive bids ceteris paribus keeping all other probabilities fixed. Formally, for $j \in B^{0}$, consider the function $f_{j}(x)$ in case all $x$ variables on which it depends are fixed, except for $x_{i}$ and $x_{i+1}$, where $i+1 \leq j-1$. Note that by fixing all other variables, the sum $x_{i}+x_{i+1}$ is also fixed. Let $b=x_{i}+x_{i+1} \in[0,1]$ and define the one-dimensional 
function $g_{i, j}:[0, b] \rightarrow[0,1]$ as

$$
g_{i, j}(a)=f_{j}\left(x_{0}, \ldots, x_{i-1}, a, b-a, x_{i+2}, \ldots, x_{j-1}, r_{j}(x)\right) .
$$

The technical condition is as follows:

Assumption 9 For all $j \in B^{0}$ and $i+2 \leq j, g_{i, j}(a)$ is strictly quasi-convex in $a \in[0, b]$.

We postpone discussing this assumption and first establish the main result of this section.

Theorem 10 Let Assumption 9 hold and $(z, x)$ is an optimal solution of (5) such that $z>0$. Then, $x \in \Delta^{N E}$ and $u(x, x)=z$ and $\sum_{i \in B^{0}} x_{i}=1$ in (5).

We prove this result in several lemmas. The first result relates any optimum of program (5) to the property of the support derived in Lemma 5.

Lemma 11 Let $(x, z)$ be an optimal solution of $(5)$ such that $z>0$. Then, there exists $a \bar{\beta} \geq 0$ such that $S^{0}(x)=\{0, \ldots, \bar{\beta}\}$.

Proof Suppose not. Then, there exists an optimal solution $(x, z)$ of $(5)$ for which there exists some $i \in B^{0} \backslash\{0\}$ such that $x_{i-1}=0$ and $x_{i}>0$. The proof consist of redistributing part of the variable $x_{i}$ over $x_{0}, \ldots, x_{i-1}$ such that a new feasible solution $\left(z^{\prime}, y\right)$ is constructed with $u(j, y)<u(j, x)$ for all $j \in B^{0}$ and $0 \leq z^{\prime}<z$.

First, choose $\varepsilon_{0}, \varepsilon_{1}, \ldots, \varepsilon_{i}>0$ such that $\sum_{m=0}^{j-1} \varepsilon_{m} \leq \varepsilon_{j}$ for all $j \leq i$ and $\varepsilon_{i}<x_{i}$. Define

$$
y_{j}=\left\{\begin{array}{ll}
x_{j}+\varepsilon_{j}, & j \leq i-1, \\
x_{j}-\varepsilon_{j}, & j=i, \\
x_{j}, & j \geq i+1,
\end{array} \quad \text { and } \quad z^{\prime}=\max \{0, u(0, y), \ldots, u(M-c, y)\}\right.
$$

Then, for all $j \in B^{0}, y_{j} \geq 0$ (with strict inequality for all $j \leq i$ ) and $\sum_{i=0}^{M-c} y_{i}=$ $\sum_{i=0}^{M-c} x_{i}+\sum_{j=0}^{i-1} \varepsilon_{j}-\varepsilon_{i} \leq 1$. Thus, $\left(y, z^{\prime}\right)$ is a feasible solution of (5).

Next, we show that $z^{\prime}<z$. We partition all $j \in B^{0}$ according to $j \geq i$ and $j \leq i-1$.

1. $j \geq i$ : By $x_{i-1}=0$, it follows for every $j \geq i$ that $F_{j} \subseteq F_{i-1}$ and, thus, $f_{j}(x) \leq f_{i-1}(x)$. Moreover, from $x_{i}>0$ it follows that $f_{i-1}(x) \geq x_{i}^{n}>0$ and, thus for all $j \geq i$ :

$$
\begin{aligned}
u(j, x) & =(M-j) f_{j}(x)-c \leq(M-j) f_{i-1}(x)-c \\
& <(M-i+1) f_{i-1}(x)-c=u(i-1, x) .
\end{aligned}
$$

Hence, $z \geq u(i-1, x)>u(j, x)$ for $j=i, i+1, \ldots, M-c$ and, in particular, $u(i, x)<z$. Since the maximal component-wise difference between $x$ and $y$ is $\varepsilon_{i}$ it follows by continuity that $u(j, y)<z$ for all $j \geq i$ if all $\varepsilon_{0}, \cdots, \varepsilon_{i}$ are small enough. To the precise specification of sufficiently small $\varepsilon_{0}, \ldots, \varepsilon_{i}$, we turn next under 2. 
2. $j \leq i-1$ : By (3), we have that the modified function $f_{j}$ only depends on the variables $x_{0}, \ldots, x_{j-1}, r_{j} \geq 0$, where $r_{j}=1-\sum_{m=0}^{j} x_{m}$. Furthermore, $x_{i}>0$ implies $\sum_{m=0}^{j} x_{m} \leq 1-x_{i}<1$ and, therefore, $r_{j}=r_{j}(x)>0$. By Lemma 8 , the modified function $f_{j}(x)$ is continuous and non-decreasing in all $j+1$ variables. Similar, $y_{0}, \ldots, y_{j-1}, r_{j}^{\prime}>0$ with $r_{j}^{\prime}=1-\sum_{m=0}^{j} y_{m}$ and, by Lemma 8 , modified function $f_{j}(y)$ is increasing in all these $j+1$ variables. Note that $y_{m}=x_{m}+\varepsilon_{m}>x_{m}$ for all $m \leq j-1$ implies $r_{j}^{\prime}<r_{j}$. Therefore, we must choose all $\varepsilon_{0}, \ldots, \varepsilon_{i}$ such that for every $j \leq i-1$ the decrease from $r_{j}$ to $r_{j}^{\prime}$ by $\varepsilon_{j}$ dominates the combined increases from $x_{m}$ to $y_{m}$ by $\varepsilon_{m}$ for all $m \leq j-1$. Since modified $f_{j}(x)$ is continuous, symmetric in $x_{0}, \ldots, x_{j-1}$ and non-decreasing $x_{0}, \ldots, x_{j-1}, r_{j}$, there exist $0<\varepsilon_{0} \ll \ldots \ll \varepsilon_{i-1} \ll \varepsilon_{i}<x_{i}$ such that $f_{j}(y)<f_{j}(x)$ for all $j \leq i-1$, where we write $a \ll b$ if $0<a<b$ and $\frac{a}{b}>0$ is arbitrarily small. Then, $u(j, y)<u(j, x)$ for all $j \in B^{0}$, including for those under 1 .

Hence, $z^{\prime}=\max _{j}\{0, u(1, y), \ldots, u(M-c, y)\}<z$ for all $j \in B^{0}$ and this contradicts that $(x, z)$ is an optimal solution of $(5)$.

Corollary 12 Let $(x, z)$ be an optimal solution of (5) such that $z>0$. Then, $\bar{\beta} \geq 1$.

Proof Suppose not, meaning $\bar{\beta}=0$. Then, $z>0$ and $\bar{\beta}=0$ imply $x_{0}=1, f_{0}(x)=0$ and $f_{i}(x)=1$ for all $i \geq 1$ meaning $u(0, x)=0$ and $z=u(1, x)=M-c-1$. By Proposition 1 and Lemma 5, the support on $B^{0}$ of any $\hat{x} \in \Delta^{N E}$ such that $\hat{z}=$ $u(\hat{x}, \hat{x})>0$ contains the subset $\{0,1\}$. Furthermore, in any symmetric NE there is a positive probability that no bidder wins the auction, i.e., $f_{i}(\hat{x})<1$ for all $i \in S(\hat{x})$. Therefore, $\hat{z}=u(1, \hat{x})<M-c-1=z$, which violates that the optimal $(z, x)$ is such that $z \in[0, \hat{z}]$. Clearly, a contradiction.

The previous result is independent of Assumption 9, but the following result also needs this assumption.

Lemma 13 Let Assumption 9 hold and $(z, x)$ is an optimal solution of (5) such that $z>0$. Then, $i \in S(x)$ implies $u(i, x)=z$.

Proof Suppose not. Then, for the optimal solution $(x, z)$ of (5) define $i \in S^{0}(x)$ as the largest $i \leq \bar{\beta}$ for which $u(i, x)<z$. Then, $x_{i+1}, \ldots, x_{\bar{\beta}}>0$ (by Lemma 11), $u(i+1, x)=\cdots=u(\bar{\beta}, x)=z$ and $u(\bar{\beta}+1, x) \leq z$. Also, $u(j, x)<z$ for all $j \geq \bar{\beta}+2$, because $x_{\bar{\beta}+1}=\ldots=x_{j-1}=0$ implies $f_{j}(x)=f_{\bar{\beta}+1}(x)$. The proof consist of redistributing part of the probability $x_{i}$ over $x_{0}, \ldots, x_{i-1}$ and $x_{i+1}$ such that a new feasible solution $\left(z^{\prime}, y\right)$ is constructed with $u(j, y)<z$ for all $j \in B^{0}$ and $0 \leq z^{\prime}<z$.

Without loss of generality, let $i<M-c$. Otherwise, $\left(z^{\prime}, y\right)$ as constructed in the proof of Lemma 11 immediately applies in case $i=M-c$ to improve upon $(z, x)$. Here, we need a different $\left(z^{\prime}, y\right)$. Choose $\varepsilon_{0}, \varepsilon_{1}, \ldots, \varepsilon_{i}, \varepsilon_{i+1}>0$ such that 
$\sum_{m=0}^{j-1} \varepsilon_{m} \leq \varepsilon_{j}$ for all $j \leq i, \varepsilon_{i}<x_{i}$ and $\varepsilon_{i+1}=\varepsilon_{i}-\sum_{j=0}^{i-1} \varepsilon_{j}$. Define $y$ such that

$$
y_{j}=\left\{\begin{array}{ll}
x_{j}+\varepsilon_{j}, & j \leq i-1, \\
x_{j}-\varepsilon_{j}, & j=i, \\
x_{j}+\varepsilon_{j}, & j=i+1 \\
x_{j}, & j \geq i+2,
\end{array} \quad \text { and } \quad z^{\prime}=\max \{0, u(0, y), \ldots, u(M-c, y)\}(.7)\right.
$$

Then, for all $j \in B^{0}, y_{j} \geq 0$ (with strict inequality for all $j \in S^{0}(x)$ and, possibly, also for $j=\bar{\beta}+1$ in case $i=\bar{\beta}$ ) and $\sum_{i=0}^{M-c} y_{i}=\sum_{i=0}^{M-c} x_{i} \leq 1$. Note that $y$ is constructed such that $r_{j}(y)=r_{j}(x)$ for all $j \geq i+1$, which is exploited below. Thus, $\left(y, z^{\prime}\right)$ is a feasible solution of (5). We consider two cases: $j \leq i+1$ and $j \geq i+2$.

1. $j \leq i+1$ : Similar as in the proof of Lemma $11,0<\varepsilon_{0} \ll \ldots \ll \varepsilon_{i-1} \ll$ $\varepsilon_{i} \ll x_{i}$ and continuity ensures $f_{j}(y)<f_{j}(x)$ for all $j=0, \ldots, i-1$ and, thus, $u(j, y)<u(j, x) \leq z$. For $j=i, f_{j}(y)>f_{j}(x)$, but for sufficiently small $\varepsilon$ 's we can ensure $u(i, y)<z$. Finally, for $j=i+1$ we have $r_{i+1}(y)=r_{i+1}(x)$ and the effect in $\varepsilon_{i}$ dominates $f_{i+1}(y)$ through $y_{i}=x_{i}-\varepsilon$. Hence, by continuity, $f_{i+1}(y)<f_{i+1}(x)$ and, thus, $u(i+1, y)<u(i+1, x) \leq z$.

2. $j \geq i+2$ : For these $j$, the issue is that $\varepsilon_{i}$ and $\varepsilon_{i+1}$ are of the same magnitude (in contrast to $\varepsilon_{m} \ll \varepsilon_{i}$ for all $\left.m \leq i-1\right)$ and $y_{i}=x_{i}-\varepsilon_{i}$ and $y_{i+1}=x_{i+1}+\varepsilon_{i+1}$ have opposite effects on $f_{j}(y)$. We have to make sure that the decrease from $x_{i}$ to $y_{i}$ dominates the increase from $x_{i+1}$ to $y_{i+1}$. We first establish the following claim.

Claim $1 x_{i+1}<x_{i}$.

The arguments in the proof of Lemma 5 apply: $x_{i} \leq x_{i+1} \Longrightarrow f_{i}(x) \geq f_{i+1}(x)$. This implies the contradiction $u(i, x)>u(i+1, x)=z$. Hence, $x_{i+1}<x_{i}$.

Next, we concentrate on $\varepsilon_{i}$ and $\varepsilon_{i+1}$ that are of the same magnitude.

Claim $2 g_{i, j}\left(x_{i}-\varepsilon_{i}\right)<f_{j}(x)$ for all $j \geq i+2$.

Consider $g_{i, j}(a)$ and note that $i \in S^{0}(x)$ implies that $b=x_{i}+x_{i+1}>0$. By Lemma $7, g_{i, j}(a)=g_{i, j}(b-a)$ for every $a \in[0, b]$. By definition of strictly quasi-convexity in the variable $a$, for every $a \in\left(0, \frac{b}{2}\right)$

$$
\begin{aligned}
g_{i, j}\left(\frac{b}{2}\right) & =g_{i, j}\left(\frac{1}{2} a+\frac{1}{2}(b-a)\right)<\max \left\{g_{i, j}(a), g_{i, j}(b-a)\right\} \\
& =g_{i, j}(a)=g_{i, j}(b-a) .
\end{aligned}
$$

Hence, $g_{i, j}$ is minimal in $a=\frac{b}{2}$ and, as a gets closer to $\frac{b}{2}, g_{i, j}(a)$ strictly decreases. Since $x_{i+1}<x_{i}$, we have that $x_{i}>\frac{b}{2}$ and, thus, $g_{i, j}\left(x_{i}-\varepsilon_{i}\right)<g_{i, j}\left(x_{i}\right)=f_{j}(x)$ for $x_{i}-\varepsilon_{i}>\frac{b}{2}$.

Claim $3 f_{j}(y)<f_{j}(x)$.

Define $y^{\prime} \in \Delta$ by $y_{i}^{\prime}=x_{i}-\varepsilon_{i}, y_{i+1}^{\prime}=x_{i+1}+\varepsilon_{i}$ and $y_{l}^{\prime}=x_{l}$ if $l \neq i, i+1$. Then, the length (norm) $\left|y-y^{\prime}\right|$ is arbitrary small compared to $|x-y|$. Thus, by continuity of the function $f_{j}$, we have that $f_{j}\left(y^{\prime}\right)=g_{i, j}\left(x_{i}-\varepsilon_{i}\right)<f_{j}(x)$ implies that $f_{j}(y)<f_{j}(x)$.

Claim 3 implies that $u(j, y)<u(j, x)$ for $j=i+2, i+3, \ldots, M-c$. 
Hence, $u(j, y)<z$ for all $j \in B^{0}$ and, thus, $z^{\prime}<z$ which contradicts that $(x, z)$ is an optimal solution of (5).

The following lemma completes the proof of Theorem 10 .

Lemma 14 Let Assumption 9 hold and $(z, x)$ is an optimal solution of (5) such that $z>0$. Then, $x \in \Delta$ is a symmetric $N E, u(x, x)=z$ and $\sum_{i \in B^{0}} x_{i}=1$ in (5).

Proof By Lemma 13, for every $i \in S^{0}(x): u(i, x)=z$. Hence, $u(x, x)=z$. Next, for every $j \in B^{0}: u(j, x) \leq z$ and, thus, for every $y \in \Delta: u(y, x) \leq z$. Hence, $x$ is a symmetric NE such that $u(x, x)=z$. Finally, we show that $x_{N}=0$. Suppose not, then $(z, x)$ is such that $x_{N}=1-\sum_{i \in B^{0}} x_{i}>0$. For $0<\varepsilon<x_{N}$, define $y \in \Delta$ such that $y_{N}=x_{N}-\varepsilon, y_{\bar{\beta}}=x_{\bar{\beta}}+\varepsilon$ and $y_{i}=x_{i}$ for all other $i \neq \bar{\beta}$. Then, for sufficiently small $\varepsilon, u(i, y)<z$ for all $i \geq \bar{\beta}$. Since also $u(i, y)=u(i, x)=z$ for all $i \leq \bar{\beta}-1$, we have that $(z, y)$ is another optimal solution in (5). Since $y$ fails the property of Lemma 13 , we arrived at a contradiction. Hence, $x_{N}=0$ implies $\sum_{i \in S^{0}(x)} x_{i}=\sum_{i \in B^{0}} x_{i}=1$ in $(5)$.

Note that the proof of Theorem 10 consists of two crucial steps, Lemma 11 and 13. The proof of Lemma 11 is independent of Assumption 9 and only require $z>0$, because otherwise the arguments would fail. In order to see this, in the case there exists an $x \in \Delta^{N E}$ such that $z=u(x, x)=0$, then $(0, x)$ is an optimal solution. Unfortunately, every $y \in \Delta$ such that $(0, y)$ is feasible in (5) is also an optimal solution and, therefore, not every such $(0, y)$ corresponds to a NE. For example, for $n+1=3$, $M=4$ and $c=1$, we obtain from Table 1 that $x \in \Delta^{N E}$ implies $u(x, x)=0$. Implementation of (5) in the optimization package GAMS ${ }^{5}$ yields the optimal solution $(z, y)$ with $z=0$ and $y=(0.0000,0.289,0.500,0.211,0.000,0.000)$, which differs from the NE $x$. Moreover, $u(1, y)=-0.244<0$ implies that $y$ fails the NE conditions. This implies that $z>0$ is necessary condition.

Lemma 13 also has an additional sufficient condition, i.e., Assumption 9. Under this assumption, the redistribution of probabilities described by $y$ in (7) improves the objective function of the program (5). It is unclear whether Assumption 9 covers the entire class of auctions considered in this paper. Verification whether $g_{i, j}(a)$ is strictly quasi-convex for all $i, j \geq i+2$, all $n+1 \geq 3$ and all $x \in \Delta$ is a Herculean task. Assumption 9 is less restrictive than assuming $f_{j}(x)$ is strictly (quasi-)convex in all $x_{0}, \ldots, x_{j-1}$. We report that strictly convex can be shown for $n+1=3,4$, all $a \in[0,1]$ and all other $x_{m} \in \mathbb{R}_{+}$, which follows directly from $g_{i, j}^{\prime \prime}(a)>0 .^{6}$ Therefore, Assumption 9 does specify a non-empty subclass of auctions. The latter approach, however, is too crude and breaks down for $n+1 \geq 5$, because then odd powers of the third degree or higher with negative coefficients might appear in $g_{i, j}^{\prime \prime}(a)$. Introducing the constraints $a \leq b=x_{0}+x_{1}$ and $x \in \Delta$ make verification of the sign of $g_{i, j}^{\prime \prime}(a)$ unworkable for arbitrary parameter values, because the function $g_{i, j}(a)$ is highly nonlinear and the support can be any subset of $S^{0}(x)$. For the same reasons, application of the necessary and sufficient conditions for additively

\footnotetext{
5 For more information on GAMS, we refer to Brooke et al. (1998).

6 Upon request, a proof is available from the authors.
} 
decomposed quasi-convex function in Crouzeix and Lindberg (1986) to (6) is also unworkable.

The optimal strategy of program (5) can also be interpreted as the maximin value of a game where a single bidder faces a bidding ring formed by all other bidders that are all restricted to follow the same bidding strategy. Then, the larger the single bidder's expected utility, the lower the ring's expected utility is, which suggests a competitive game. Therefore, by minimizing the single bidder's expected utility the ring gives away the least expected amount. By symmetry of all bidders in the auction, the ring's minimizing bidding strategy is also one of the single bidder's best responses to this minimizing bidding strategy. Therefore, if $(z, x)$ solves (5) such that $z>0$, then $(x, x) \in \arg \max _{x^{\prime} \in \Delta} \min _{x^{\prime \prime} \in \Delta} u\left(x^{\prime}, x^{\prime \prime}\right)$. By von Neumann's famous maximin equals minimax theorem, we also have that $(x, x) \in$ $\arg \min _{x^{\prime \prime} \in \Delta} \max _{x^{\prime} \in \Delta} u\left(x^{\prime}, x^{\prime \prime}\right)$.

The results obtained thus far have several implications (under Assumption 9):

1. If all symmetric NE have positive expected NE utilities, then (5) identifies the symmetric NE with the lowest expected NE utilities and, consequently, $z>0$.

2. If $(z, x)$ is an optimal solution and $z>0$, then this rules out any symmetric NE with expected $\mathrm{NE}$ utilities equal to 0 .

3. If $(z, x)$ is an optimal solution and $z=0$, then there exists a symmetric NE with expected NE utilities of 0 (because the condition under point 1 cannot hold).

These implications make program (5) very interesting for numerical analyses, because this program can answer the question whether for certain parameter values the expected NE utilities are positive or zero and, if positive, it yields a symmetric NE.

\section{Comparative statics of symmetric NE}

In this section, we perform comparative statics with respect to the key parameters such as large numbers of bidders, large bidding cost and large values of the item. In order to state this result, we define $\Delta^{N E}(M, c, n+1)$ as the set of symmetric NE for $M, c$ and $n+1$.

Theorem 15 If the number of bidders is sufficiently large, such as $n+1 \geq \frac{M}{c}$, or the cost of making a bid are sufficiently large, such as $c \geq \max _{x \in \Delta^{N E}}(M, 1, n+1) f_{0}(x) M$, then in any symmetric NE non-participating has a positive probability and the expected NE payoffs are zero. In particular, as the number of bidders goes to infinity, the probability of non-participating goes to one. For sufficiently large value of the item, all bidders participate for sure and expect to make a positive gain. Moreover, as the value goes to infinity, the highest bid in the support also goes to infinity.

The intuition behind Theorem 15 is quite intuitive, an increased probability of participation deteriorates the probability of winning and, therefore, erodes the profitability of entering. If too many bidders enter, then the entire expected surplus vanishes. Indeed, when the number of potential bidders goes to infinity, the individual probability of participating becomes negligible and the remaining probabilities over bids 
all converge to zero. Similar, if the cost of bidding become too high, these costs cannot be recovered by the expected gains of winning and bidders retreat by increasing their probability of non-participating. Whenever, the probability of non-participating is positive, the expected NE payoffs are zero. In that case, we arrive at a result similar to the classic result known for markets with endogenous entry: under a fixed market entrance fee, potential producers enter as long as there are expected net profits from doing so are larger than the entrance fee. In the symmetric NE of the auction, costly bidding erodes expected positive net gains until these expected gains are equal to the payoff of non-participating. The value of the item has a different effect compared to the previous two parameters: if the value becomes large enough, all bidders will participate and expect to make a profit. Moreover, a higher value make higher bids more attractive and, therefore, increases the support. In particular, if the value goes to infinity, the support becomes unbounded.

We now turn our attention to the proof of Theorem 15. Theorem 15 is shown by three major lemma's, each involves one of the three key parameters, that also provide some additional insights. First, we consider large numbers of bidders.

Lemma 16 Let $x \in \Delta^{N E}(M, c, n+1)$. For any $n+1 \geq \frac{M}{c}$, then $x_{N}>0$ and $u(x, x)=0$. If $n+1$ goes to infinity, then $x_{N}$ goes to 1 .

Proof For arbitrary $n+1$ and $x \in \Delta^{N E}(M, c, n+1)$, define $p(x ; n+1) \leq \frac{1}{n+1}$ as the probability that bidder 1 wins the auction. Note that the positive probability that nobody wins the auction, which is at least $x_{0}^{n+1}>0$, yields $p(x ; n+1)<\frac{1}{n+1}$. As $n+1$ goes to $\infty$, this probability converges to 0 . Suppose for all $n+1$ that $x_{N}(n+1)=0, x(n+1) \in \Delta^{N E}(M, c, n+1)$. Then, the expected NE payoff is at most $p(x ; n+1) M-c<\frac{1}{n+1} M-c$. The upper bound converges to $-c$ as $n+1$ goes to $\infty$. Negative NE payoffs contradict Proposition 1 . Therefore, for large enough $n+1, x_{N}>0$. Since $p(x ; n+1)<\frac{1}{n+1}$, bidder 1 would certainly make a loss if the upper bound $\frac{1}{n+1} M-c$ becomes non-positive, which yields the lower bound on $n+1$. Finally, for all $n+1 \geq \frac{M}{c}, u(x, x)=0=M f_{0}(x)-c$ implies that $x_{0}=1-\left(\frac{c}{M}\right)^{\frac{1}{n}} \rightarrow 0$ as $n+1$ goes to $\infty$. By Lemma $5, x_{0}$ is the largest probability in $S^{0}(x)$ and, therefore, all probabilities in $S^{0}(x)$ converge to 0 . Hence, in the limit $x_{N}=1$.

The lower bound on the number of bidders is derived from the intuition that the probability of winning the auction should yield a sufficiently large expected revenue to cover the cost of making a bid. In case, all bidders enter the auction for sure, the probability of winning would converge to 0 if the number of bidders would go to infinity, which means that, in the case of too many bidders, non-participating becomes a profitable deviation. Note that this intuition provides a sufficient condition for $x_{N}>0$, i.e., however, not necessary because it is derived under overestimation of the true probability of winning by comparing it to a standard lottery. Note that the lower bound can be rewritten as $c \geq \frac{M}{n+1}$, which is also the condition for zero expected NE utilities in Proposition 2 . Since the true probability of winning will be strictly smaller than in a lottery and winning positive bids have to be paid, it is possible $x_{N}>0$ also holds for some $n+1$ just below $\frac{M}{c}$. This statement is illustrated by Table 4 of Sect. 7, where all off diagonal elements have an expected NE utility of zero. Therefore, for given $M$ 
and $c$, we may only expect symmetric NE with positive expected NE payoffs for sufficiently small $n+1<\frac{M}{c}$. As the number of bidders approaches infinity, the probability that an individual bidder enters becomes negligible. The limit probability distribution over the numerical bids has limit probabilities of zero. Under a large Poisson game with bid cost of zero and the winner does not have to pay the winning bid, Östling et al. (2007) also derive a uniform limit distribution over numerical bids in, but with positive probabilities. This result is fundamentally different to our result where it is non-participating that prevails in the limit when bidding cost are positive.

The second lemma puts an upper bound upon the cost for positive expected NE payoffs.

Lemma 17 For any $x(1) \in \Delta^{N E}(M, 1, n+1)$ such that $u(x(1), x(1))=$ $f_{0}(x(1)) M-1 \geq 0$ and all $c \leq f_{0}(x(1)) M$ itholds that $x(1) \in \Delta^{N E}(M, c, n+1)$. Moreover, for $c>1, x(c) \in \Delta^{N E}(M, c, n+1)$ such that $u(x(c), x(c))=$ $f_{0}(x(c)) M-c>0$ implies that $x(c) \in \Delta^{N E}(M, 1, n+1)$.

Proof Consider $x \in \Delta^{N E}(M, 1, n+1)$. By Lemma 4, for all $i \in S^{0}(x)$ and $j \in B^{0}$ :

$(M-i) f_{i}(x)-1 \geq(M-j) f_{j}(x)-1 \Leftrightarrow(M-i) f_{i}(x)-c \geq(M-j) f_{j}(x)-c$,

and equality holds if also $j \in S^{0}(x)$. Therefore, different $c$ do not affect these inequalities. Then, $x$ is also a NE for $c$ provided that the expected utility under $c$, which is $f_{0}(x) M-c$, obeys the non-negativity implied by Proposition 1. Starting with arbitrary $c \geq 1$ yields the second statement, where the condition $f_{0}(x(c)) M-c>0$ ensures that $S(x(c))=S^{0}(x(c))$ and $x_{0}(\hat{c})<1-\left(\frac{\hat{c}}{M}\right)^{\frac{1}{n}}$ for all $\hat{c}=1, \ldots, c$ by (4).

Lemma 17 is based upon the observation that the equilibrium conditions for numeric bids in $B^{0}$ are invariant with respect to the cost of making a bid. Therefore, as long as the expected payoffs of participating for sure under $x(1) \in \Delta^{N E}(M, 1, n+1)$ under different cost $c$ outweigh the opportunity cost of non-participating, participation by playing $x$ (1) remains a NE. This invariance cannot hold for any NE $x(c) \in$ $\Delta^{N E}(M, c, n+1)$ with a positive probability of non-participating, i.e., $x_{N}(c)>0$, because then $u(x, x)=0$ implies that $x_{0}(c)=1-\left(\frac{c}{M}\right)^{\frac{1}{n}}$ depends upon $c$. Moreover, the probability $x_{0}(c)$ is decreasing in $c$. Therefore, $x(c) \in \Delta^{N E}(M, c, n+1)$ such that $x_{N}(c)>0$ implies $x(c) \notin \Delta^{N E}\left(M, c^{\prime}, n+1\right)$ for $c^{\prime} \neq c$. A similar invariance also fails for $M$ and $M^{\prime} \neq M$. Therefore, $x \in \Delta^{N E}(M, c, n+1)$ implies $x \notin \Delta^{N E}\left(M^{\prime}, c, n+1\right)$.

The third lemma states that for large enough values $M$, all bidders participate for sure and expect to make a profit.

Lemma 18 For $c$ and $n+1$ fixed, let $\{x(M)\}_{M=1}^{\infty}$ be an infinite sequence of $x(M) \in \Delta^{N E}(M, c, n+1)$. If $\lim \sup _{M \rightarrow \infty} x_{k}(M)=0$ for some $k \in \mathbb{N}_{0}$, then $\lim _{M \rightarrow \infty} x_{i}(M)=0$ for all $i \in \mathbb{N}_{0}$.

Proof For $i \geq k$, we have by Lemma 5 that $0 \leq x_{i}(M) \leq x_{k}(M)$ for every $M$. Thus, $\lim _{M \rightarrow \infty} x_{i}(M)=\limsup _{M \rightarrow \infty} x_{k}(M)=0$. For $k=0$ the proof is finished and we 
therefore consider $k \geq 1$. Then, it suffices to prove that

$$
\lim _{M \rightarrow \infty} x_{k-1}(M)=0
$$

because the lemma then follows by induction. Establishing (8), requires:

Claim 19 For any $\delta>0$, we have $\lim \sup _{M \rightarrow \infty} x_{k-1}^{M} \leq \delta$.

By definition of $\lim \sup _{M \rightarrow \infty} x_{k}(M)=0$, it follows that for every $\varepsilon>0$ there exists some $N(\varepsilon) \in \mathbb{N}$ such that $x_{k}(M)<\varepsilon$ for every $M \geq N(\varepsilon)$. Given $\delta>0$, we choose $\varepsilon=\frac{\delta^{n}}{n}$. Note that for every $x(M) \in \Delta^{N E}(M, c, n+1)$, bid $k$ is a unique-lowest bid if bid $k-1$ is a lowest-unique bid and none of the other $n$ bidders submit bid $k$. Moreover, bid $k$ would also be a lowest-unique bid if all other $n$ players bid $k-1$. Combining this gives for all $M \geq N(\varepsilon)$ the inequality

$f_{k}(x(M)) \geq f_{k-1}(x(M))-\operatorname{Pr}($ at least one other of $n$ players bids $k)+\left(x_{k-1}(M)\right)^{n}$ and, thus, for all $M \geq N(\varepsilon)$, we have

$$
f_{k}(x(M)) \geq f_{k-1}(x(M))-n \varepsilon+\left(x_{k-1}(M)\right)^{n} .
$$

Combining (9) with Lemma 5 and Lemma 6 gives for every $M \geq N(\varepsilon)$ that

$$
\begin{aligned}
& (M-k)\left(f_{k-1}(x(M))-n \varepsilon+\left(x_{k-1}(M)\right)^{n}\right) \leq(M-k) f_{k}(x(M)) \\
& =u_{k}(x(M))+c \leq u_{k-1}(x(M))+c=(M-k+1) f_{k-1}(x(M)),
\end{aligned}
$$

and, thus,

$$
(M-k)\left(\left(x_{k-1}(M)\right)^{n}-n \varepsilon\right) \leq f_{k-1}(x(M)) \leq \frac{1}{n+1}
$$

Hence,

$$
\left(x_{k-1}(M)\right)^{n} \leq \frac{1}{(n+1)(M-k)}+n \varepsilon,
$$

and, thus,

$$
\begin{aligned}
x_{k-1}(M) & \leq\left[\frac{1}{(n+1)(M-k)}+n \varepsilon\right]^{1 / n} \leq\left[\frac{1}{(n+1)(M-k)}\right]^{1 / n}+[n \varepsilon]^{1 / n} \\
& =\left[\frac{1}{(n+1)(M-k)}\right]^{1 / n}+\delta .
\end{aligned}
$$

From this, it follows that $\lim \sup _{M \rightarrow \infty} x_{k}(M) \leq \delta$ for every $\delta>0$, which establishes the claim. From the claim, it follows that for any $\delta>0$ :

$$
0 \leq \liminf _{M \rightarrow \infty} x_{k-1}(M) \leq \limsup _{M \rightarrow \infty} x_{k-1}(M) \leq \delta,
$$


and, thus,

$$
0=\liminf _{M \rightarrow \infty} x_{k-1}(M)=\limsup _{M \rightarrow \infty} x_{k-1}(M)=\lim _{M \rightarrow \infty} x_{k-1}(M) .
$$

This establishes (8) and completes the proof of the lemma.

These results imply the following:

Corollary 20 For c and $n+1$ fixed, let $\{x(M)\}_{M=1}^{\infty}$ be an infinite sequence of $x(M) \in$ $\Delta^{N E}(M, c, n+1)$. Then, for every bid $i \in \mathbb{N}_{0}$, we have that $\lim \sup _{M \rightarrow \infty} x_{i}(M)>0$.

Proof For fixed $n+1$, we have by (4) that $\lim _{\sup } \rightarrow \infty x_{0}(M) \geq 1-\left(\frac{1}{n+1}\right)^{1 / n}>0$. This implies the implication of Lemma 18 does not hold and, hence, the if cannot hold either. Therefore, for every $i \in \mathbb{N}_{0}: \lim _{\sup _{M \rightarrow \infty}} x_{i}(M)>0$.

Corollary 21 For $c$ and $n+1$ fixed, there does not exist a finite integer $\bar{\beta} \in \mathbb{N}$, such that for all values $M \in \mathbb{N}$, there exists an $x(M) \in \Delta^{N E}(M, c, n+1)$ for which it holds that $x_{i}(M)=0$ for all bids $i>\bar{\beta}, i \in \mathbb{N}$.

Informally speaking, the last result states an impossibility result for bounded supports. In order to put it differently, the size of the support of symmetric NEs is unbounded when the value $M$ goes to infinity. In the case of a unique symmetric NE for all $M$, the infinite sequence $\{x(M)\}_{M=1}^{\infty}$ of NEs in Lemma 18 is uniquely determined and $\lim _{M \rightarrow \infty} x_{i}(M)$ exists for every bid $i \in \mathbb{N}_{0}$. Then, Corollary $20 \mathrm{im}$ plies that each limit is strictly positive, i.e., $\lim _{M \rightarrow \infty} x_{i}(M)>0$ for every $i \in \mathbb{N}_{0}$. Since every player will submit a bid for sure when $M$ is sufficiently large, expected NE payoffs are positive and $x_{N}=0$. By Lemma 17, the NE strategies become invariant with respect to cost $c$ and this implies that $\lim _{M \rightarrow \infty} x_{i}(M)$ only depends on $i \in \mathbb{N}_{0}$ and the number of players $n+1$.

A final remark concerns the case of costless bidding, i.e., $c=0$, implemented in laboratory experiments. Then, in any $x \in \Delta^{N E}$, we have $u(0, x)=M f_{0}(x)>0$ and, hence, $u(x, x)>0$ and $x_{N}=0$ (all bidders participate for sure). Since Lemma 17 also extends to this case, our invariance result implies that these equilibria also remain NE under relatively low cost $c<u(x(0), x(0)), c \in \mathbb{N}$. Hence, for appropriate parameters $n+1$ and $M$, there is theoretically no loss of generality by implementing a lowest-unique sealed-bid auction with low bidding cost $c$ in a laboratory experiment as such auction with $\operatorname{cost} c=0$. In doing so, however, one foregoes the opportunity to test the theoretical invariance result and whether observed entry is consistent with theory. Also, implementing $c=0$ in the laboratory foregoes testing situations with non-trivial entry decisions, i.e., sufficiently large $c$ such that $x_{N}>0$.

\section{Numerical analysis of symmetric NEs}

The previous sections establish that the functional form of the winning probabilities in (3) obstructs a full characterization of symmetric NEs. In order to obtain additional insights, we resort to three numerical methods. First, we further explore Gambit. 
Second, we implement the optimization program (5) in the numerical optimization package GAMS. Third, simulation of the replicator dynamics in Maple ${ }^{7}$ to discuss the announced topic of evolution stability of bidding behavior.

In Gambit, we computed all NE and implemented a sufficiently large subset of $B$. This slowed down the computations considerably in computing NEs when $n+1$ and $M$ increase. Since also the implementation in Gambit is time consuming, we restricted the use of this package to $n+1=3$ or 4 and parameter values $M$ in the range of 3 to 25 and $c=1$. Gambit always reported a single symmetric NE. A fast and efficient way to compute the symmetric NE under Gambit is to compute the quantal response equilibrium, see McKelvey and Palfrey (1995). They also show that the quantal response equilibrium may fail to be evolutionary stable and, therefore, we cannot conclude evolutionary stability from applying this equilibrium concept.

Program (5) is implemented in GAMS and, for all parameter values run, GAMS reports normal completion. For $n+1 \geq 8$, our computer program becomes very slow due to the need to compute a large amount of multinomial coefficients prior to the numerical optimization. For that reason, we did not perform any computations in GAMS for $n+1 \geq 10$.

Next, we briefly introduce the replicator dynamics. Bidders in the lowest-unique sealed-bid auction come from a single population and any vector in $\Delta$ represents the population fractions of bids in this population. Time $t \in \mathbb{R}_{+}$is continuous and $x(t) \in \Delta$ represents the population fractions at time $t$, where $x(0)$ represents the initial population fractions. We assume that the population size $N \gg n+1$ such that modifications due to finite populations can be neglected. The replicator dynamics are defined as in Weibull (1995) and given by the following system of differential equations:

$$
\dot{x}_{b}(t)=x_{b}(t)[u(b, x(t))-u(x(t), x(t))], \quad b \in B .
$$

In Bukowski and Miekisz (2004), it is shown that the set of evolutionary stable strategies (ESS) is a subset of the set of asymptotically stable equilibrium (ASE) strategies, and that the latter is a subset of the set of NE. Formally, $\Delta^{E S S} \subseteq \Delta^{A S E} \subseteq \Delta^{N E}$. Verification of the definition of ESS for the lowest-unique sealed-bid auction or of the optimum of program (5) is too difficult given that we cannot fully characterize NEs or this optimum. Recall that quantal response equilibrium may not be ESS. For that reason, we resort to numerically solving for stationary distributions of (10). In the case of convergence, numerical methods for simulating time paths of replicator dynamics (10) yield ASE strategies, and this is as close as we (numerically) can get to ESS. All numerical simulations were performed in the software package Maple by invoking the fourth-order Runga-Kutta method with a step size of 0.01, see e.g., Betounes (2001). The initial population fractions were chosen as the uniform distribution over a large support of consecutive numbers containing 0 . In all simulations, the initial support $S(x(0)) \supset S\left(x^{*}\right)$, where $x^{*}=\lim _{t \rightarrow \infty} x(t)$. The replicator dynamics converged in all parameter values run, but convergence slows down considerably for $n+1 \geq 8$.

For $n+1=3$ or 4 , we first computed all NEs with Gambit for several parameter values $3 \leq M \leq 25$ and $c=1$. Then, we ran the replicator dynamics and obtained

\footnotetext{
7 For more information on Maple, we refer to www.maplesoft.com.
} 
Table 2 Symmetric NEs for several values of $M, n+1$, and $c=1$

\begin{tabular}{|c|c|c|c|c|c|c|c|c|}
\hline$n+1$ & $M$ & $x_{0}$ & $x_{1}$ & $x_{2}$ & $x_{3}$ & $x_{4}$ & $x_{5}$ & $x_{6}$ \\
\hline \multirow[t]{4}{*}{3} & 50 & 0.464 & 0.258 & 0.148 & 0.091 & 0.040 & & \\
\hline & 100 & 0.460 & 0.253 & 0.142 & 0.083 & 0.057 & 0.005 & \\
\hline & 200 & 0.458 & 0.251 & 0.139 & 0.079 & 0.047 & 0.026 & \\
\hline & 400 & 0.457 & 0.250 & 0.137 & 0.076 & 0.044 & 0.028 & 0.007 \\
\hline \multirow[t]{4}{*}{4} & 50 & 0.452 & 0.428 & 0.119 & & & & \\
\hline & 100 & 0.450 & 0.427 & 0.122 & 0.000 & & & \\
\hline & 200 & 0.449 & 0.426 & 0.124 & 0.001 & & & \\
\hline & 400 & 0.448 & 0.425 & 0.125 & 0.001 & & & \\
\hline \multirow[t]{4}{*}{5} & 50 & 0.364 & 0.320 & 0.193 & 0.095 & 0.028 & & \\
\hline & 100 & 0.361 & 0.318 & 0.192 & 0.096 & 0.033 & & \\
\hline & 200 & 0.359 & 0.316 & 0.192 & 0.095 & 0.034 & 0.003 & \\
\hline & 400 & 0.359 & 0.316 & 0.191 & 0.095 & 0.035 & 0.004 & \\
\hline \multirow[t]{4}{*}{6} & 50 & 0.331 & 0.301 & 0.234 & 0.118 & 0.016 & & \\
\hline & 100 & 0.329 & 0.299 & 0.233 & 0.120 & 0.019 & & \\
\hline & 200 & 0.328 & 0.298 & 0.232 & 0.122 & 0.020 & & \\
\hline & 400 & 0.327 & 0.298 & 0.232 & 0.122 & 0.021 & 0.000 & \\
\hline \multirow[t]{4}{*}{7} & 50 & 0.299 & 0.274 & 0.228 & 0.140 & 0.054 & 0.005 & \\
\hline & 100 & 0.297 & 0.272 & 0.226 & 0.140 & 0.056 & 0.008 & \\
\hline & 200 & 0.296 & 0.271 & 0.226 & 0.141 & 0.057 & 0.010 & \\
\hline & 400 & 0.295 & 0.271 & 0.225 & 0.141 & 0.058 & 0.010 & \\
\hline \multirow[t]{4}{*}{8} & 50 & 0.275 & 0.255 & 0.220 & 0.157 & 0.078 & 0.014 & \\
\hline & 100 & 0.273 & 0.253 & 0.219 & 0.157 & 0.080 & 0.017 & \\
\hline & 200 & 0.272 & 0.252 & 0.218 & 0.157 & 0.081 & 0.019 & 0.000 \\
\hline & 400 & 0.272 & 0.252 & 0.218 & 0.157 & 0.082 & 0.019 & 0.001 \\
\hline \multirow[t]{4}{*}{9} & 50 & 0.256 & 0.238 & 0.211 & 0.165 & 0.098 & 0.031 & 0.000 \\
\hline & 100 & 0.254 & 0.237 & 0.210 & 0.165 & 0.099 & 0.034 & 0.003 \\
\hline & 200 & 0.253 & 0.236 & 0.209 & 0.164 & 0.100 & 0.035 & 0.004 \\
\hline & 400 & 0.252 & 0.235 & 0.209 & 0.164 & 0.100 & 0.036 & 0.004 \\
\hline
\end{tabular}

convergence to the symmetric NE. In case $x \in \Delta^{N E}$ such that $u(x, x)>0$, we also performed the numerical optimization algorithms in GAMS. This was done to check the numerical accuracy of the limit solution of the replicator dynamics. Since GAMS returned approximately $\left(u\left(x^{*}, x^{*}\right), x^{*}\right)$, all limit solutions $x^{*}$ of the replicator dynamics are very accurate and $\left(u\left(x^{*}, x^{*}\right), x^{*}\right)$ is feasible in (5). In all these cases, all three numerical methods provide consistent and reinforcing results.

Table 2 reports several numerical solutions obtained from both (5) and the replicator dynamics (10), where 0.000 indicates a positive probability that is insignificant. Blank spaces indicate a solution with at least 7 digits equal to 0 that, therefore, we presume are zero. 
Table 3 Symmetric NEs for several values of $M, n+1$, and $c=1$

\begin{tabular}{|c|c|c|c|c|c|c|c|c|c|c|}
\hline$n+1$ & $M$ & $x_{N}$ & $x_{0}$ & $x_{1}$ & $x_{2}$ & $x_{3}$ & $x_{4}$ & $x_{5}$ & $x_{6}$ & $x_{7}$ \\
\hline \multirow[t]{5}{*}{4} & $10^{3}$ & & 0.4480 & 0.4251 & 0.1253 & 0.0016 & & & & \\
\hline & $10^{4}$ & & 0.4478 & 0.4249 & 0.1256 & 0.0017 & & & & \\
\hline & $10^{5}$ & & 0.4477 & 0.4249 & 0.1257 & 0.0017 & & & & \\
\hline & $10^{6}$ & & 0.4477 & 0.4249 & 0.1257 & 0.0017 & & & & \\
\hline & $10^{7}$ & & 0.4477 & 0.4249 & 0.1257 & 0.0017 & & & & \\
\hline \multirow[t]{5}{*}{6} & $10^{3}$ & & 0.3268 & 0.2977 & 0.2315 & 0.1223 & 0.0214 & 0.0003 & & \\
\hline & $10^{4}$ & & 0.3266 & 0.2975 & 0.2314 & 0.1225 & 0.0216 & 0.0004 & & \\
\hline & $10^{5}$ & & 0.3266 & 0.2975 & 0.2314 & 0.1225 & 0.0216 & 0.0005 & & \\
\hline & $10^{6}$ & & 0.3266 & 0.2975 & 0.2314 & 0.1225 & 0.0216 & 0.0005 & & \\
\hline & $10^{7}$ & & 0.3266 & 0.2975 & 0.2314 & 0.1225 & 0.0216 & 0.0005 & & \\
\hline \multirow[t]{5}{*}{8} & $10^{3}$ & & 0.2712 & 0.2514 & 0.2178 & 0.1571 & 0.0821 & 0.0196 & 0.0008 & \\
\hline & $10^{4}$ & & 0.2710 & 0.2512 & 0.2176 & 0.1571 & 0.0822 & 0.0199 & 0.0010 & \\
\hline & $10^{5}$ & & 0.2712 & 0.2512 & 0.2176 & 0.1571 & 0.0822 & 0.0199 & 0.0010 & \\
\hline & $10^{6}$ & & 0.2712 & 0.2512 & 0.2176 & 0.1571 & 0.0822 & 0.0199 & 0.0010 & 0.0000 \\
\hline & $10^{7}$ & & 0.2712 & 0.2512 & 0.2176 & 0.1571 & 0.0822 & 0.0199 & 0.0010 & 0.0000 \\
\hline
\end{tabular}

The support of the symmetric NE grows if either $M$ increases or the number of bidders $n+1$ increases under fixed $c$ and this is in accordance with Theorem 15. More bidders increase the competition for the single item for sale and bidders spread out their bids to seek profitable opportunities. However, for small numbers of bidders, roughly the range $n+1 \leq 6$, Table 2 shows non-monotonic behavior with respect to the size of the support. For $n+1=3$, this can be explained by the fact that the highest bid wins if the other two bidders make the same (lower) bid. In the case of $n+1=4$, the highest bid can only win if the other three bidders all make the same bid, which is less likely then two bidders making the same bid. Therefore, the higher bids are less attractive and the support retracts. For $n+1=5$, the probability of winning with the highest bid is somewhat better again, because it also wins, e.g., if two opponents both bid some lower bid and the other two opponents both bid some other lower bid. When $n+1$ increases further, these kind of odd/even considerations lose significance and, then, the numerical examples suggest that the numerical support is non-decreasing in $n+1$.

Table 3 represents simulation results for increasingly large values of the item $M$ for $n+1$ equal to 4,6 , and 8 . The change in probabilities and the growth of the support is very slow. For $n+1=4$, this table might give the false impression that there is convergence to a finite support, but according to Theorem 15 , this is not the case.

Table 4 reports parameter values with zero and positive expected utilities. As $n+1$ increases, the consecutive set of low values $M$ for which the expected NE utilities are 0 grows. If the sufficient condition $n+1 \geq \frac{M}{c}$ in Theorem 15 for zero profits would also be a necessary condition, then the entire lower triangular matrix would be 
Table 4 Parameter values for which the expected NE utilities are either positive $(+)$ or zero $(0)$ under $c=1$

\begin{tabular}{lllll}
\hline$\frac{M}{c}$ & $n+1$ & & & \\
\cline { 2 - 5 } & 3 & 4 & 5 & 6 \\
\hline 3 & 0 & 0 & 0 & 0 \\
4 & 0 & 0 & 0 & 0 \\
5 & + & 0 & 0 & 0 \\
6 & + & 0 & 0 & 0 \\
7 & + & 0 & 0 & 0 \\
8 & + & + & + & 0 \\
9 & + & + & + & + \\
\hline
\end{tabular}

filled with +'s. Since it is not, the sufficient condition is only a rough indication for the parameter values for which the expected NE utilities are zero.

In order to summarize, Gambit returns a single symmetric NE for $n+1=3$ and 4 . Furthermore, the replicator dynamics always converge to a unique limit distribution over $B$, i.e., a symmetric NE. ${ }^{8}$ Moreover, if the ASE has positive expected NE utilities, then this limit solution is one-to-one related to the optimal solution of program (5). This gives numerical evidence for the following conjecture.

Conjecture 22 The lowest-unique sealed-bid auction has a unique symmetric NE that is also ASE. If $x \in \Delta^{N E}$ and $u(x, x)>0$, then $(u(x, x), x)$ is an optimal solution of (5).

One final remark is in place. The replicator dynamics always converges, in particular, if $u(x, x)=0$. Under $u(x, x)>0$, computing limit solutions in Maple takes considerably more time than optimization in GAMS. Therefore, we regard simulating (10) and program (5) as two complementary approaches in numerically computing the symmetric NE.

\section{Concluding remarks}

The lowest-unique sealed-bid auction is a non-trivial auction to analyze, and it fails a full characterization because the winning probabilities involves summing orders of large degrees. In contrast, the lowest-bid auction has a simple solution, trivial comparative statics, and allows straightforward numerical implementation. Despite the many technical non-trivialities, we derive general properties of symmetric NEs, perform comparative statics, and a mathematical program that provides a maximin interpretation to the (conjectured) unique symmetric NE.

The extension to multiple bids is analyzed in Eichberger and Vinogradov (2008). Generally speaking, their main result states that, for some endogenous consecutive sets of bids that contains the lowest bid, bidders randomize over all consecutive subsets (of this endogenous set) that contain the lowest bid. In other words, single bids do not arise endogenously from the multiple-bids model. This implies that the single bid model requires an analysis on its own, as is done in this article.

\footnotetext{
8 Note that symmetric games might fail an ASE or admit multiple ASEs.
} 
In Östling et al. (2007) and Rapoport et al. (2007), laboratory experiments are implemented by imposing zero bidding cost, which ensures trivial endogenous entry. Other than its easy implementation, this assumption is not motivated, and has as a serious drawback that it removes entry decisions from the experiment. Our invariance result provides an underpinning for this simplifying assumption: if expected NE payoffs are positive under zero bidding cost, then this NE also remains NE under relatively low cost. Therefore, sufficiently small bidding cost would imply a strategically equivalent experiment and may therefore be neglected. Two remarks are in place. First, by implementing both zero and small positive cost, our results provide an extra test by testing for invariance across experiments. Second, implementing experiments with relatively larger bidding cost allows for testing the more realistic endogenous entry decision under zero expected equilibrium payoffs. For example, during 2005 and 2006 in The Netherlands ran, the nationwide TV show SHOP4NOP (translated "shop for free") that auctioned items valued at most $1,000 €$ and each bid by SMS costs $70 €$ cents. This auction has zero expected NE payoffs for sure if it attracts a regular audience of $n+1 \geq 1,429$, which is $<0.01 \%$ of the population in The Netherlands.

Acknowledgments We are grateful to Quan Wen and Gerard van der Laan for valuable suggestions.

Open Access This article is distributed under the terms of the Creative Commons Attribution Noncommercial License which permits any noncommercial use, distribution, and reproduction in any medium, provided the original author(s) and source are credited.

\section{References}

Becker, J., \& Damianov, D. (2006). On the existence of symmetric mixed strategy equilibria. Economics Letters, 90, 84-87.

Betounes, D. (2001). Differential equations: Theory and applications with Maple. Berlin: Springer.

Brooke, A., Kendrick, D., Meeraus, A., \& Raman, R. (1998). Gams: A users guide. Available at the GAMS Development Corporation web site at http://www.gams.com.

Bukowski, M., \& Miekisz, J. (2004). Evolutionary and asymptotic stability in symmetric multi-player games. International Journal of Game Theory, 33, 41-54.

Crouzeix, J.-P., \& Lindberg, P. (1986). Additively decomposed quasiconvex functions. Mathematical Programming, 35, 42-57.

Eichberger, J., \& Vinogradov, D. (2008). Least unmatched price auctions. Heidelberg: University of Heidelberg, Mimeo.

Houba, H., van der Laan, D., \& Veldhuizen, D. (2008). The lowest-unique sealed-bid auction. TI Discussion Paper 08-049, Tinbergen Institute, Amsterdam/Rotterdam. Available at www.tinbergen.nl.

Krishna, V. (2002). Auction theory. San Diego: Academic Press.

McKelvey, R., \& Palfrey, T. (1995). Quantal response equilibria for normal form games. Games and Economic Behavior, 10, 6-38.

McKelvey, R., Richard, D., McLennan, A., \& Turocy, T. (2006). Gambit: Software tools for game theory, version 0.2006.01.20. http://econweb.tamu.edu/gambit.

Myerson, R. (1998). Population uncertainty and poisson games. International Journal of Game Theory, $27,375-392$.

Myerson, R. (2000). Large poisson games. Journal of Economic Theory, 94, 7-45.

Östling, R., Wang, J., Chou, E., \& Camerer C. (2007). Field and lab convergence in poisson lupi games. SSE/EFI working paper series in Economics and Finance 671. 
Rapoport, A., Otsubo H., Kim B., \& Stein W. (2007). Unique bid auctions: Equilibrium solutions and experimental evidence. Discussion Paper, University of Arizona.

Raviv, Y., \& Virag G. (2008) Gambling by auctions. International Journal of Industrial Organization, 27, 369-378.

Weibull, J. (1995). Evolutionary game theory. Cambridge: MIT Press. 